\title{
Extracellular Formation of Silver Nanoparticles by the Cell Filtrate of Actinomyces spp. NSX-333
}

\author{
Kh. G. Ganbarov', N. A. Agayeva ${ }^{2}$, M. A. $\operatorname{Ramazanov}^{3}$, S. M. Abdulhamidova ${ }^{1}$, \\ Z. A. Agamaliyev ${ }^{3}$, G. M. Eyvazova ${ }^{3}$ \\ ${ }^{1}$ Department of Microbiology, Baku State University, Baku, Azerbaijan. \\ ${ }^{2}$ Department of Microbiology and Immunology, Azerbaijcan Medical University, Baku, Azerbaijan. \\ ${ }^{3}$ Department of Physical Chemistry of Nanomaterials, Baku State University, Baku, Azerbaijan
}

\begin{abstract}
In this paper, we have used a biological method of the biosynthesis of silver nanoparticles, using supernatant (culture liquid) of bacterium Actinomyces spp.NSX-333,, isolated from Azerbaijani soil.The culture liquid has been obtained by culturing the bacterium at $28^{\circ} \mathrm{C}$ for 5-6 days. It was showed that during the incubation of the culture liquid with $\mathrm{AgNO}_{3}$ solution, a restoration of silver ions takes place and stable silver nanoparticlesare formed. Initially, the nanoparticle formation process was visually observed by a color change of the incubation solution.The nanoparticles have characterized using a UV spectrophotometer, anenergy dispersive X-ray spectroscopy (EDXS) and a scanning electron microscope (SEM).UV spectrophotometric analysis of nanoparticlesshowed the presence of absorption in the range of $410 \mathrm{~nm}$, which is characteristic of silver nanoparticles. EDXS spectrum showed a peak corresponding to the silver nanoparticles. Obtained silver nanoparticles had a spherical shape and size in the range of 20 to $130 \mathrm{~nm}$. Clusters of nanoparticles were also observed.
\end{abstract}

Keywords: Actinomyces spp.NSX-333, culture fluid, silver nanoparticles, UV spectroscopy, EDXA spectroscopy, SEM.

\section{INTRODUCTION}

One of the fastest growing areas of modern nanotechnology at the present time is the creation and use of nanoparticles of various materials. Special attention has recently drawn to the silver nanoparticles, which are characterized by unique properties. These properties are associated with a high ratio of surface to volume, which determines greater efficiency of their action.Much attention is paid to the functional activity of silver nanoparticles from the standpoint of imparting both bactericide and bacteriostatistic properties of various materials and products. They have an extremely large specific surface area.This increases the area of the silver contact with bacteria or viruses, greatly improving its destructive action for microorganisms $[5,7,8,11]$.

Thus, the use of silver as a nanoparticle allowshundreds of times to bring down the concentration of silver storing all bactericidalproperties.Silver nanoparticles are used as a biocide additive - in the form of a modifier. This modifier is destined for creation and production of new materials, coatings and other products with biocide properties of a wide range of activities. Choice of silver nanocomposites for impregnation is due to their significant and indisputable advantages over all existing antimicrobial remedies.With a wide range of antimicrobial activity, silver compoundsare, in many ways, devoid of the disadvantages that are related to the problem of resistance of pathogen microorganisms to them $[9$, 20,22].

A bunch of physical and chemical methods have been developed for the synthesis of nanoparticles of suitable size and shape. However, in spite of their successful applicationthey often remain expensiveand require hazardous chemical compounds. Therefore,there is a need to develop effective methods of synthesis, different nanoparticles using biological objects, particularly, microorganisms safe for the environment and humans [6, 10,21].

At the present time an active search for effective microorganisms (bacteria, yeast, andmoldy fungi) is conductedto produce silver nanoparticles [4, 4, 12-14,18]. A series of works are devoted to the biosynthesis of silver nanoparticles using branched bacteria (Streptomyces rochei, S.species, S.albidoflavus, S. aurefacies) [1, 2, 15-17, 19]. 
At the present work branched bacterium Actinomyces spp.NSX-33 3isolated from Azerbaijani environment has been used for synthesis of silver nanaoparticles.

\section{Materials ANd Methods}

Bacterial strain Actinomyces spp.NSX-333, isolated from Azerbaijani soil, was used as the object.Pure culture bacteria were maintained for further researchesin a mineral medium of the following composition (g / 1): soluble starch -20; $\mathrm{K}_{2} \mathrm{HPO}_{4}-0,05 ; \mathrm{KNO}_{3}-0,1 ; \mathrm{FeSO}_{4}-0,005 ; \mathrm{MgSO}_{4}-0,05$; distilled water-1litre; at $4-6^{\circ} \mathrm{C}$.

To study the ability of bacterial strain to form silver nanoparticles, culture was grown aerobicallyin $250 \mathrm{ml}$ Erlenmeyer flask, containing $100 \mathrm{ml}$ of mineral medium with the above composition by adding yeast extracts in an amount of $2 \mathrm{~g} / \mathrm{l}$.

Liquid medium was inoculated with the culture of Actinomyces spp.NSX-333 and incubated at $30^{\circ} \mathrm{Cwithin}$ 5-7 dayson a rotary shaker.Then the biomass was separated by filtration through a filter paper and the culture liquid was used for the formation of nanoparticles.Culture fluid in an amount of $50 \mathrm{ml}$ was mixed with $50 \mathrm{ml}$ of $1 \mathrm{mM} \mathrm{AgNO}$ and was incubated at $30^{\circ} \mathrm{C}$ in dark conditions within 72-120 hours.Culture fluidwithout added $\mathrm{AgNO}_{3}$ was used as a control.

Initially, nanoparticle formation was observed by the color darkening of the incubation mixture. The optical properties of the nanoparticles were investigated by the spectrophotometer "Specord 250 plus" (Germany). Morphological characteristics and the size of the silver nanoparticles were studied by the scanning electron microscope (SEM) "JEOLJSM-7600F" (Japan).Energy-Dispersive X-rays analysis( EDXA)) of the the obtained nanaoparticles was used as well.

\section{RESUltS AND DiscuSSIONS}

By the incubation of bacterial culture fluid Actinomyces spp.NSX-333with $1 \mathrm{mM}$ solution of $\mathrm{AgNO}_{3}$ within 72-129 hours, we observe the darkening of the incubation mixture to a reddish-brown, which is characteristic for silver nanoparticles (fig. 1).

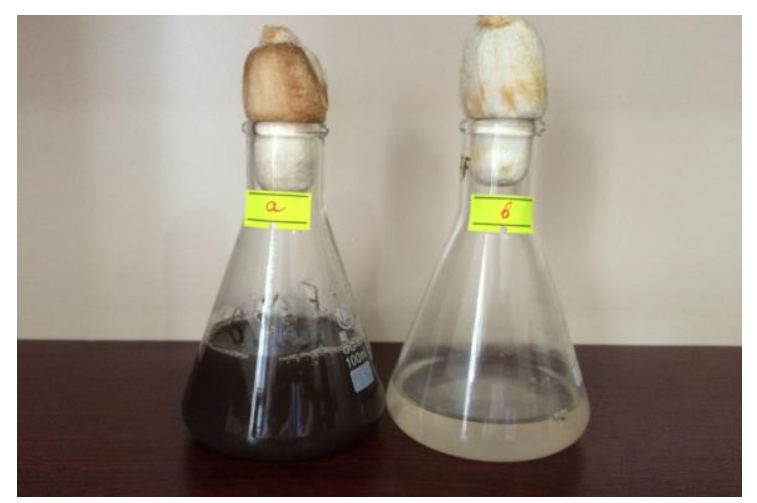

Figure1. Color change of reaction mixture during formation of silver nanoparticles byActinomyces spp.NSX333: a-experiment, b-control

UV spectrophotometric analysis of nanoparticlesshowed the presence of absorption in the range of $410 \mathrm{~nm}$ for 7 hours, which is characteristic of silver nanoparticles (fig.2).

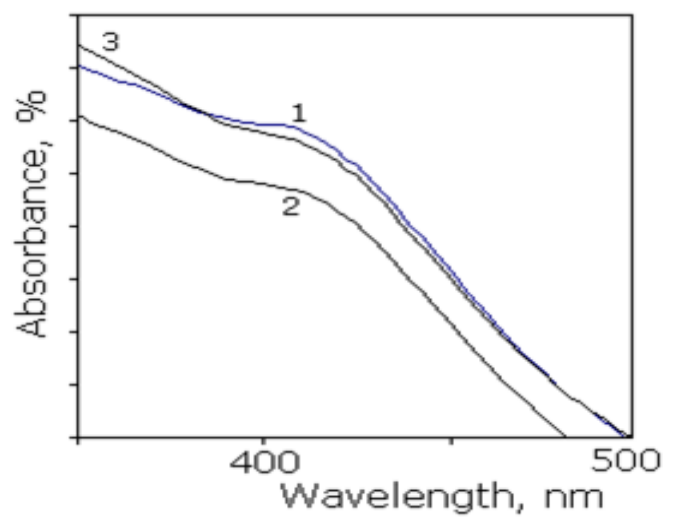

Figure2. UV spectr of silver nanoparticles synthesized by Actinomyces spp.NSX-333 
The morphology and shape of silver nanoparticles were studied by scanning electron microscopy SEM method (fig.3). Have been found that the sizes of nanoparticles distribute in the range of 20-130 $\mathrm{nm}$. In ouropinion the widesize distributionis explained with uneven agglomeration of silver clusters in the suspension.

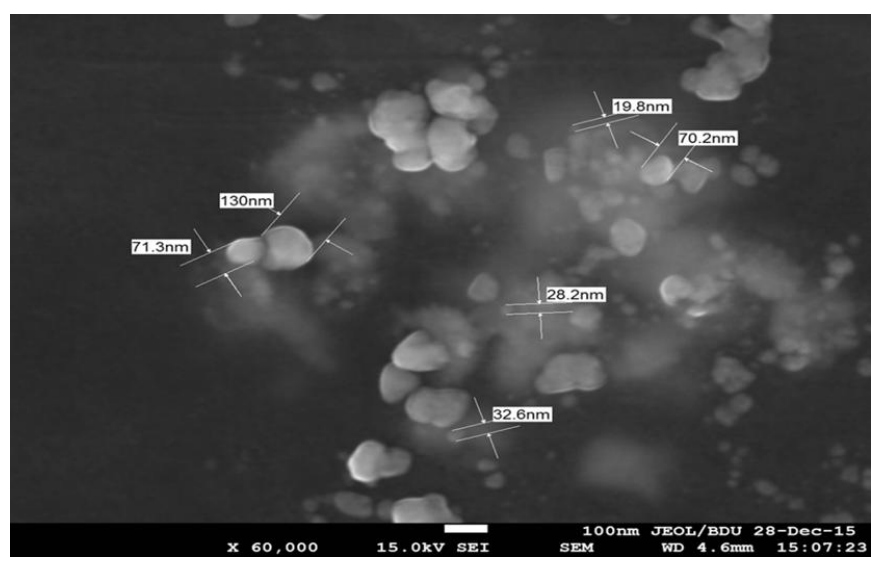

Figure3. SEM images of silver nanoparticles synthesized by Actinomyces spp.NSX-333

Fig.4 shows the Energy-Dispersive X-ray spectral analysis of obtained nanoparticles' sample and mapping of selected area for the elements distribution. As we can see from the mapping and spectral analysis the presence of peaks $\mathrm{AgLa} 1$ and $\mathrm{AgU}$ proved the presence of silver nanoparticles.

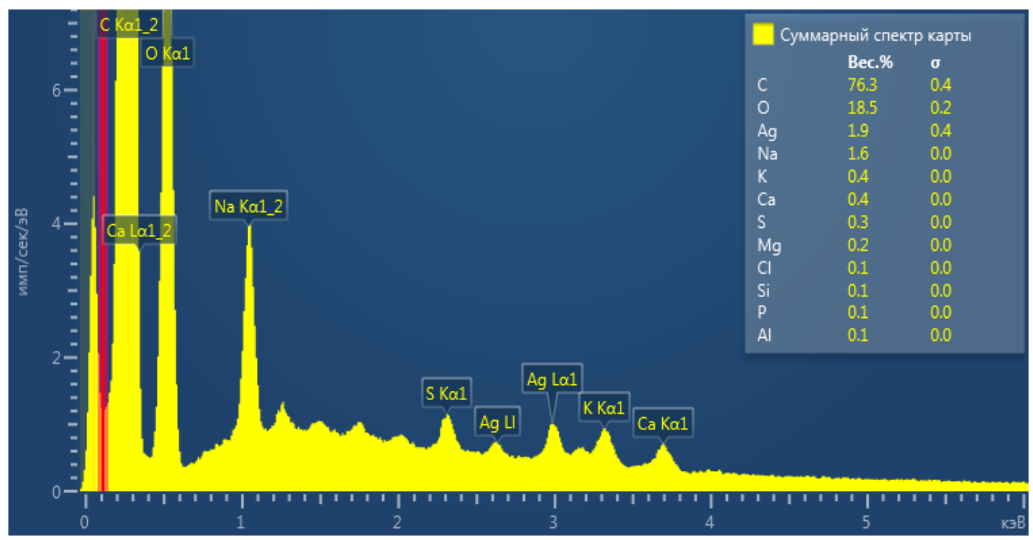

Figure4. Energy-Dispersive X-rayspectr of silver nanoparticles synthesized byActinomyces spp.NSX-333 The figure 5 shows the mapping the elements of silver nanoparticles in the solution. From this figure it is clear seen the forming of silver nanoparticles.
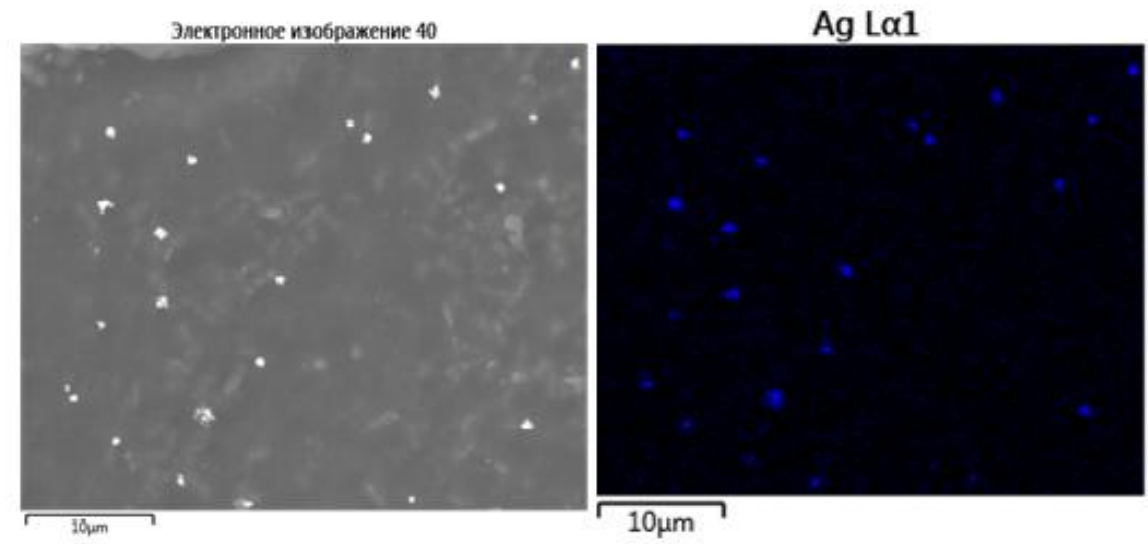

Fig5. Mapping the elements

\section{Conclusion}

Theobtainedresults enable to come to a conclusion, that bacterial culture Actinomyces spp.NSX-333is able to form silver nanoparticles extracellularly.Nanoparticles were characterized by using of UV spectrophotometer, EDXA spectrophotometer and scanning electron microscope (SEM). Silver nanoparticleshave a spherical form, and their sizevaries in the range of 20 to $130 \mathrm{~nm}$. 


\section{REFERENCES}

[1] Duron, N., Marcato P., Alves O., Gabriel I., Souza D., Esposito E. Extracelluar biosynthesis of silver nanoparticles using culture supernatant of Streptomyces species. Jour. Nanobiotechnol. 3: $1-8(2005)$

[2] Faghri Z.N., Salovti M. Extracellular biosynthesis of silver nanoparticles using cell filtrate of Streptomyces sp. ER1-3. Scientia. Transactions F: Nanotechnol 18(6): 1631-1635 (2011)

[3] GanbarovKh.G., Ahmadov I.S., Ramazanov M.A., Musayev E.M., Eyvazova G.S., Aghamaliyev Z.A. Silver nanoparticles synthesized by the Azerbaijanianevrironmental isolated AspergillusnIger. Jour. Microbiol. Biotechnol. Food Science.4(2): 137-141(2014)

[4] GanbarovKh.G., Cafarov M.M., Haciyeva F.T., Huseynova S.I., Bozkurt H.J., Ramazanov M.A.,Agamaliyev Z.A., Eyvazova G.I., Ahmadov I.S. Mycogenic formation of silver nanoparticlis by the Azerbaijanese environmental isolate Candida macedoniensis BDU-MI44. Int.Jour.ReserchStudies in Bioscien. 4(5): 1-5 (2016)

[5] Kim J., Kuk E, Yu K.,Park S., Lee H., Antimicrobial effects of silver nanoparticles. Nanomed.Nanotechnol. Biol.Med.3: 15-21(2007)

[6] Krumov N, Perner-Nochta I, Order S., Gatcheva V., Angelov A.,Posten C. Production of inorganic nanoparticles by microorganisms. Chem.Eng.Technol.32: 1026-1035 (2009)

[7] Li X., Ku H., Chen Zh., Chen G. Biosynthesis of nanoparticles by microorganisms and their applications (review article) Jour. Nanomaterials ID 270974:16 p. 2011 (DOI:10.1155/ 2011/ 270974)

[8] Mohanpuria P., Rana N., Yadov S. Biosynthesis of nanoparticles: technological concepts and future applications. Jour.Nanopart. Research.10 (3): 507-517 (2008)

[9] Mornes J.,Eleehiguerra J., Camacha A., Holt K., Kouri J., Ramirez J.,Yacaman M. The bactericidal effect of silver nanoparticles. Nanotech.16: 2346-2353 (2005)

[10] Natarajan, K. Microbial Production of Silver Nanoparticle / K.Natarajan, S.Selvaraj, V. Rama chandran Murti. Digest J. Nanomat. Biostr.5: 135-140 ( 2010)

[11] Rai M., Yadav A., Gade A. Silver nanoparticles as a new generation of antimicrobials. Biotechnology Advances, 27: 76-83 (2009)

[12] Sadowski, Z., Maliszewska I., Polowezyk I.,Kozlecki T., Grochowalska B. Biosynthesis of colloidal nanoparticles using microorganisms. Polish Jour.Chem. 82: 377-382 (2008)

[13] SadowskiZygmunt. Biosynthesis and application of silver and gold Nanoparticles.Wroclaw University of Technology 11: 257-266(2010)

[14] Sastry, M.,Ahmad A., KhanM.I. and KumarR. Biosynthesis of metal nanoparticles using fungi and actinomycete.Curr. Science 85 (2): 162-170 (2003)

[15] Selvakumaz T.,Viveka S., Praxash S., Jasmiebeaula, Uloganathan R. Antimicrobial activity of extracellularly synthesized silver nanoparticles from marincdeivedStreptomyces rochei. Intern. J.Prarma, Bioscien.3(3): 188-197(2012)

[16] Shetty P., Kumaz B., Kumar Y., Shankar G. Characterization of silver nanoparticles synthesized by using marine isolate Streptomyces albidoflavus. Jour.Microbiol.Biotechnol.22(5): 614-621 (2012)

[17] Shirley A., Dayanad B., Sreedhar B., Dastager S. Antimicrobial activity of silver nanoparticles synthesized from novel Streptomyces species. Dig.Jour.Nanomatter.Biostruc.5: 447-451 (2010)

[18] Sing P., Raya R. Biological synthesis and characterization of siver nanoparticles using the fungus Tricchodermaharzianum. Asian.Exp.Biol.Sci.2: 600-605 (2011)

[19] Vengadesh, P.K., Sundaramoorthi C., Devarasu S. Biosynthesis of silver nanoparticles from Streptomyces aureofaciens. Jour. Pharmacy Research.4(3): 820-822 (2011)

[20] Veeranna S. Burhanuddin A., Khanum Sh., Narayan S., PratimaKh. Biosynthesis and antibacterIal activity of silver nanoparticles. Reserch Jour. Biotechnol.8(2): 11-17 (2013)

[21] Vithiya,K., Sen S. Biosynthesis of nanoparticles. International Journal of Pharmaceutical Sciences and Research.2 (11): 2781-2785(2011)

[22] Zhang L., Gu F., Chan J., Wang A., Langer R., Farokhzad O. Nanoparticles in medicine: Therapeutic applications and developments.Clin.Pharmacol.Ther 83: 761-769 (2008) 